\title{
Focus on Lipids: High-Density Lipoprotein Cholesterol and Its Associated Lipoproteins in Cardiac and Renal Disease
}

\author{
Hyun Joon Shin ${ }^{a, c}$ Peter A. McCullougha, b \\ a Baylor University Medical Center, Baylor Heart and Vascular Institute, Baylor Jack and Jane Hamilton Heart and \\ Vascular Hospital, Dallas, Tex., ${ }^{\text {b}}$ The Heart Hospital, Plano, Tex., and ' Harvard School of Public Health, \\ Boston, Mass., USA
}

\section{Key Words}

Lipids · High-density lipoprotein cholesterol - Lipoproteins . ApoL1

\begin{abstract}
High-density lipoprotein cholesterol (HDL-C) contains dozens of apoproteins that participate in normal cholesterol metabolism with a reliance on renal catabolism for clearance from the body. The plasma pool of HDL-C has been an excellent inverse predictor of cardiovascular events. However, when HDL-C concentrations have been manipulated with the use of niacin, fibric acid derivatives, and cholesteryl ester transferase protein inhibitors, there has been no improvement in outcomes in patients where the low-density lipoprotein cholesterol has been well treated with statins. Apolipoprotein L1 (APOL1) is one of the minor apoproteins of HDL-C, newly discovered in 1997. Circulating APOL1 is a 43-kDa protein mainly found in the HDL3 subfraction. In patients with chronic kidney disease (CKD), mutant forms of APOL1 have been associated with rapidly progressive CKD and end-stage renal disease (ESRD). Because mutant forms of APOL1 are more prevalent in African Americans compared to Caucasians, it may explain some of the racial disparities seen in the pool of patients with ESRD in the United States. Thus, HDL-C is an important lipoprotein carrying apoproteins that play roles in vascular and kidney disease.

(c) 2014 S. Karger AG, Basel
\end{abstract}

\section{Introduction}

Elevated high-density lipoprotein cholesterol (HDLC) has been suggested to be strongly related to decreased atherosclerotic events in large population-based studies [1]. Each $1 \mathrm{mg} / \mathrm{dl}$ increase in HDL-C was associated with a decrease of $2-3 \%$ in the risk of coronary heart disease events [1]. In observational studies and early randomized trials, largely in statin-naive patients, with nicotinic acid, fibrates, and lifestyle changes, an increase in HDL-C was associated with a modest reduction in cardiovascular events (fig. 1). Recently completed studies have shed light on the relationships between the plasma pool HDL-C concentration and cardiovascular events as well as the unique effects of the apoproteins carried by the HDL particle on end-organ disease.

\section{Trials on Raising HDL-C and Clinical Outcomes}

There have been many clinical trials testing the effect of raising HDL-C. We summarize here the results of modern cholesteryl ester transfer protein (CETP) inhibi-

Changing Paradigms in Acute Kidney Injury: From Mechanisms to Management - Proceedings of the 5th Annual UAB-UCSD O'Brien Center Symposium (San Diego, Calif., USA, March 4, 2014).

\section{KARGER}

E-Mail karger@karger.com

www.karger.com/nec
(C) 2014 S. Karger AG, Basel

$1660-2110 / 14 / 1274-0158 \$ 39.50 / 0$
Peter A. McCullough, MD, MPH

Baylor Heart and Vascular Institute 621 N. Hall St., H030

Dallas, TX 75226 (USA)

E-Mail peteramccullough@gmail.com 
Fig. 1. Minor changes in HDL-C in prospective studies and reduction of cardiovascular events. Each $1 \mathrm{mg} / \mathrm{dl}$ increase in HDL-C was associated with a decrease of $2-3 \%$ in the risk of coronary heart disease (CHD) events in observational studies and early randomized trials. FHS $=$ Framingham Heart Study; LRCF = Lipid Research Clinics Prevalence Mortality Follow-Up Study; CPPT = Lipid Research Clinics Coronary Primary Prevention Trial; MRFIT = Multiple Risk Factor Intervention Trial. Reproduced from Cardenas et al. [16] and Gordon et al. [1] with permission from Circulation.

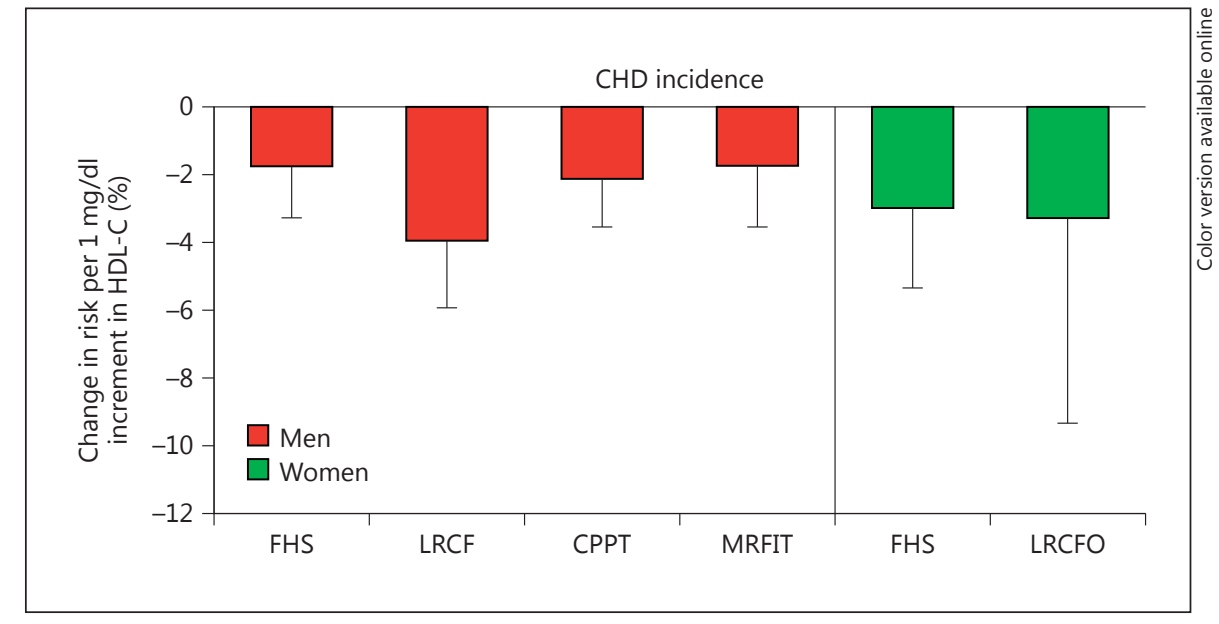

tor trials and niacin trials which have been largely carried out under a more modern standard of care where patients have control of low-density lipoprotein cholesterol (LDLC) levels through an improved diet, statins, ezetimibe, bile acid sequestrants, and other agents. These trials give a more accurate picture of the impact of raising HDL-C as an intervention on top of good clinical practice in primary or specialty care.

\section{CETP Inhibitor Trials}

CETP works to shuttle cholesterol and triglycerides between HDL and very low-density lipoprotein (VLDL) and other apolipoprotein B100-containing particles (fig. 2). Early studies demonstrated that inhibition of this protein markedly raised the HDL-C concentration; however, it was unclear whether this improved or potentially worsened the reverse cholesterol transport. The Investigation of Lipid Level Management to Understand Its Impact in Atherosclerotic Events (ILLUMINATE) trial is a phase 3 randomized double-blind clinical trial that investigated the role of the first CETP inhibitor, torcetrapib, on cardiovascular events and death in 15,067 patients at high cardiovascular risk. The patients in the torcetrapib group showed an increase of $72 \%$ in HDL cholesterol after 12 months compared to baseline [2]. However, the total mortality (HR 1.58; 95\% CI 1.14-2.19) and cardiovascular events (HR 1.25; 95\% CI 1.09-1.44) also increased significantly [2]. The off-target effect of torcetrapib of increasing the systolic blood pressure, sodium, and aldosterone was blamed as a possible mechanism that resulted in increased mortality rates and cardiovascular events $[2,3]$.
In this study, despite the overall findings, those who achieved the highest HDL-C concentrations had the lowest overall event rates.

The dal-OUTCOMES trial is a phase 3 randomized double-blind clinical trial that investigated the role of the second CETP inhibitor, dalcetrapib, on the composite primary endpoint of death from coronary heart disease, cardiac arrest with resuscitation, nonfatal myocardial infarction, unstable angina, or ischemic stroke in 15,871 patients with recent acute coronary syndrome [3]. The patients in the dalcetrapib group showed an increase of $31-40 \%$ in HDL cholesterol compared to baseline [3]. Dalcetrapib did not lower the risk of the primary endpoint (HR 1.04; 95\% CI $0.93-1.56$ ) or total mortality [3].

Anacetrapib is the third CETP inhibitor currently under evaluation in a phase 3 clinical trial (Randomized Evaluation of the Effects of Anacetrapib through Lipid Modification; REVEAL). The trial has an enrollment of 30,000 patients with atherosclerotic disease on statin treatment (http://clinicaltrials.gov/ct2/show/ NCT01252953?term\%BCreveal\&rank\%BC1).

The results of the trial on the effects of anacetrapib on the risk of myocardial infarction, coronary death, and coronary revascularization should be available in January 2017 [4].

Evacetrapib is the fourth CETP inhibitor that is now also being evaluated in a phase 3 clinical trial (Assessment of Clinical Effects of Cholesteryl Ester Transfer Protein Inhibition with Evacetrapib in Patients at a High-Risk for Vascular Outcomes; ACCELERATE) enrolling 12,000 patients with high-risk vascular disease on statin treatment (http://clinicaltrials.gov/ct2/show/record/ 


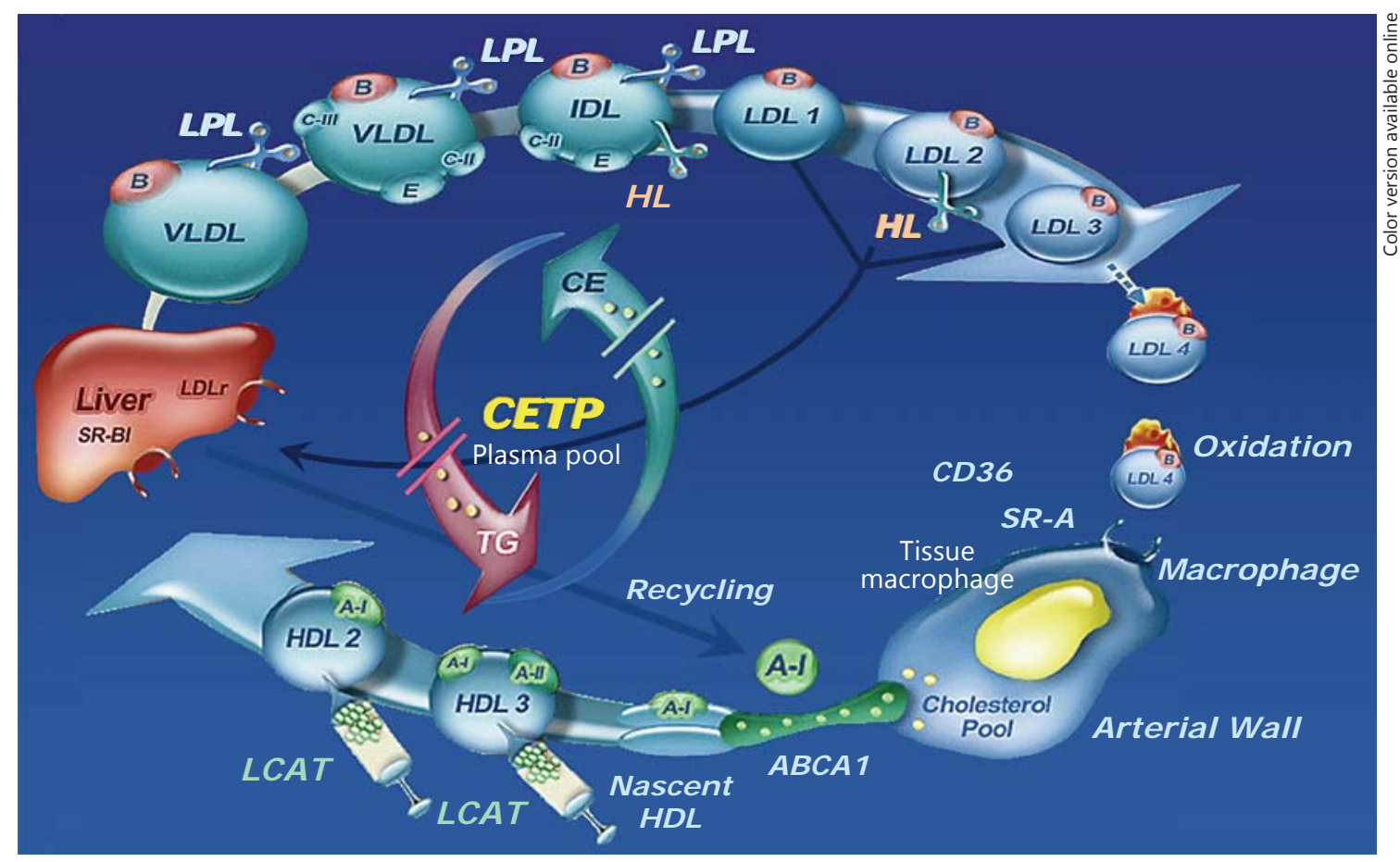

Fig. 2. Shuttling of cholesterol and triglycerides by CETP. CETP works to shuttle cholesterol and triglycerides between HDL and VLDL and other apolipoprotein B100-containing particles. ABCA1 = Adenosine triphosphate-binding cassette protein A1 transporter; $\mathrm{A} 1=$ apolipoprotein $\mathrm{A}-\mathrm{I} ; \mathrm{A} 2=$ apolipoprotein $\mathrm{A}-2$; A3 = apolipoprotein A-3; B = apolipoprotein B; C-II = apolipoprotein C-II; C-III = apolipoprotein C-III; CD36 = scavenger receptor $\mathrm{CD} 36$; $\mathrm{CETP}=$ cholesteryl ester transfer protein; $\mathrm{CE}=$ cholesteryl

NCT01687998). The results of this trial on the effects of evacetrapib on the composite endpoint of risk of myocardial infarction, cardiovascular death, stroke, hospitalization for unstable angina, and coronary revascularization will be available in January 2016 [4]. Figure 3 displays the CTEP inhibitors and summarizes their effects on HDL-C and LDL-C.

\section{Niacin Trials}

The Atherothrombosis Intervention in Metabolic Syndrome with Low HDL/High Triglycerides: Impact on Global Health Outcomes (AIM-HIGH) trial is a randomized double-blind clinical trial that investigated the effect of the addition of extended-release niacin to intensive statin therapy on the composite primary endpoint of death from coronary heart disease, nonfatal myocardial infarction, ischemic stroke, hospitalization for an ester; $\mathrm{E}=$ apolipoprotein $\mathrm{E} ; \mathrm{HL}=$ hepatic lipase; IDL = intermediate-density lipoproteins; LCAT = lecithin-cholesterol acyltransferase; $\mathrm{LDL}=$ low-density lipoproteins; $\mathrm{LDLr}=\mathrm{LDL}$ receptor; $\mathrm{LPL}=$ lipoprotein lipase; SR-A = scavenger receptor-A; SR-BI = scavenger receptor-BI; TG = triglyceride; $\mathrm{VLDL}$ = very-low-density lipoproteins. Modified from Brewer and Santamarina-Fojo [17] with permission from the American Journal of Cardiology. acute coronary syndrome, or symptom-driven coronary or cerebral revascularization in 3,414 patients with cardiovascular disease [5]. Extended-release niacin increased the HDL cholesterol from 35 to $42 \mathrm{mg} / \mathrm{dl}$ after 2 years compared to baseline [5]. Extended-release niacin did not change the risk of the primary endpoint (HR 1.02 ; 95\% CI 0.87-1.12), and the trial was stopped prematurely due to a lack of benefit and because there was a higher rate of ischemic stroke in patients in the niacin group (fig. 4) [5].

The Heart Protection Study 2-Treatment of HDL to Reduce the Incidence of Vascular Event (HPS-2THRIVE) trial is a randomized placebo-controlled trial that investigated the effect of the addition of extendedrelease niacin plus laropiprant (ERN/LRPT) to intensive statin therapy on the composite primary endpoint of cardiovascular death, myocardial infarction, stroke, or revascularization in 25,673 patients with occlusive arterial disease [6]. ERN/LRPT increased the HDL-C by $16.9 \%$ 
Fig. 3. Chemical structures of CETP inhibitors and their approximate effects on HDL-C and LDL-C. Adapted from von Eckardstein. http://www.medscape. org/noscan/slideshow/760297\#25.
Fig. 4. Main results of the AIM-HIGH trial. Extended-release niacin did not change the risk of the primary endpoint compared to the placebo group. Adapted from Boden et al. [7]. MI = Myocardial infarction. With permission from the New England Journal of Medicine.
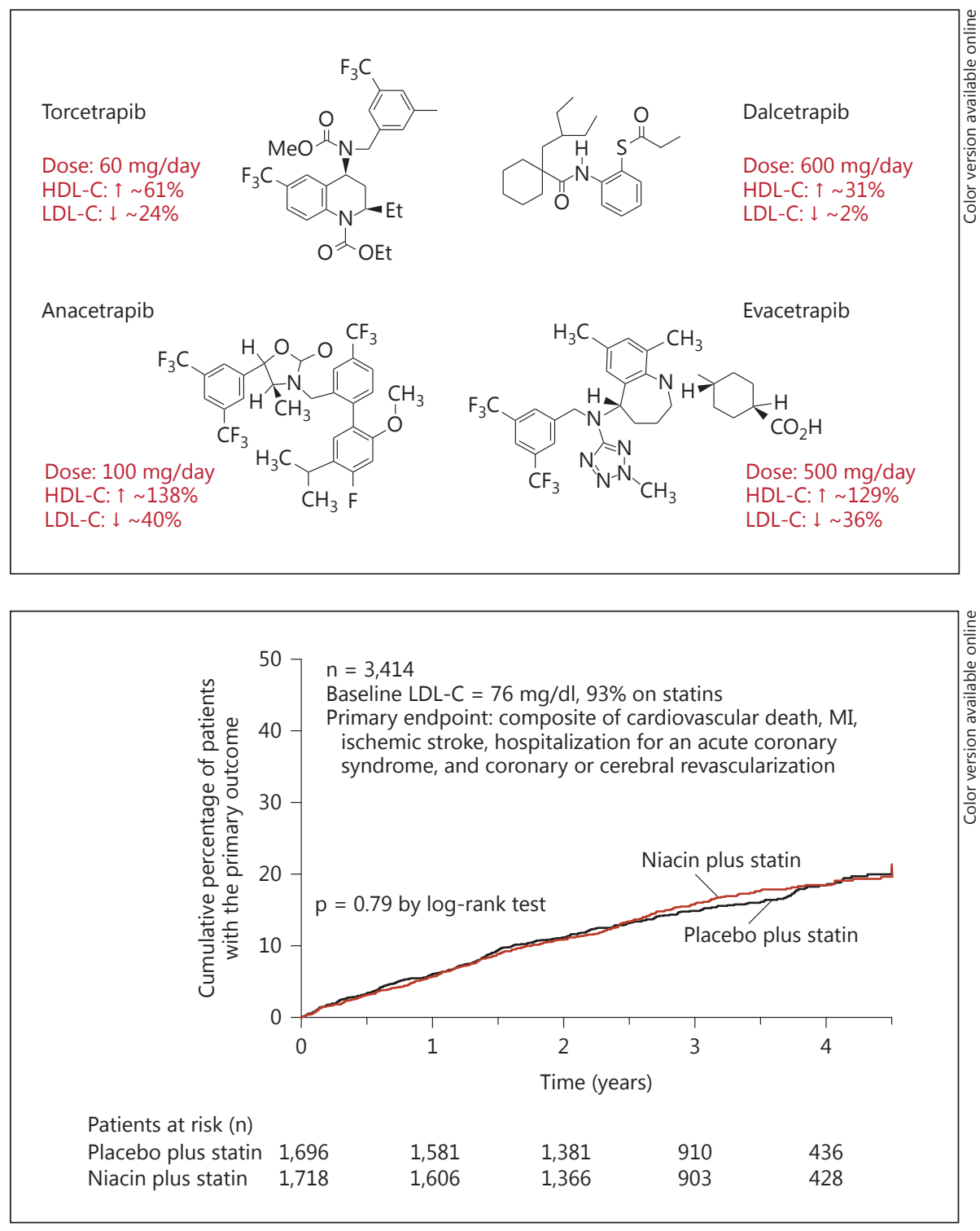

compared to baseline [6]. ERN/LRPT did not change the risk of the primary endpoint (14.5\% of the ERN/LRPT group vs. $15 \%$ of the placebo group, $p=0.29$ ) after a mean of 3.9 years of follow-up [5]. Twenty-five percent of patients in the ERN/LRPT group compared to $17 \%$ of the placebo group stopped taking the study medications because of diverse side effects including skin problems and gastrointestinal, musculoskeletal, and diabetes-related side effects and myopathy [7].

There are several possible explanations for the failure of pharmacologic elevation of HDL-C to improve the clinical outcomes in modern trials. First, it may be difficult to show an incremental benefit of raising HDL-C for

HDL-C and Its Associated Lipoproteins in Cardiac and Renal Disease the prevention of cardiovascular events when patients are currently receiving high-quality medical treatment, including intensive statin, antiplatelet, $\beta$-blocker, and angiotensin-converting enzyme inhibitor drugs $[3,5]$. Secondly, increased HDL-C may not induce the desired reverse cholesterol transport or that mechanism may be counterbalanced by another proatherosclerotic force. Only $40 \%$ of the total variance of the cholesterol efflux capacity from macrophages (a metric of HDL reflecting a reverse cholesterol transport) was explained by the HDL$\mathrm{C}$ level, so quantitative levels of HDL-C may not reflect the desired reverse cholesterol transport function adequately [8]. Also, under oxidative stress, HDL-C can 


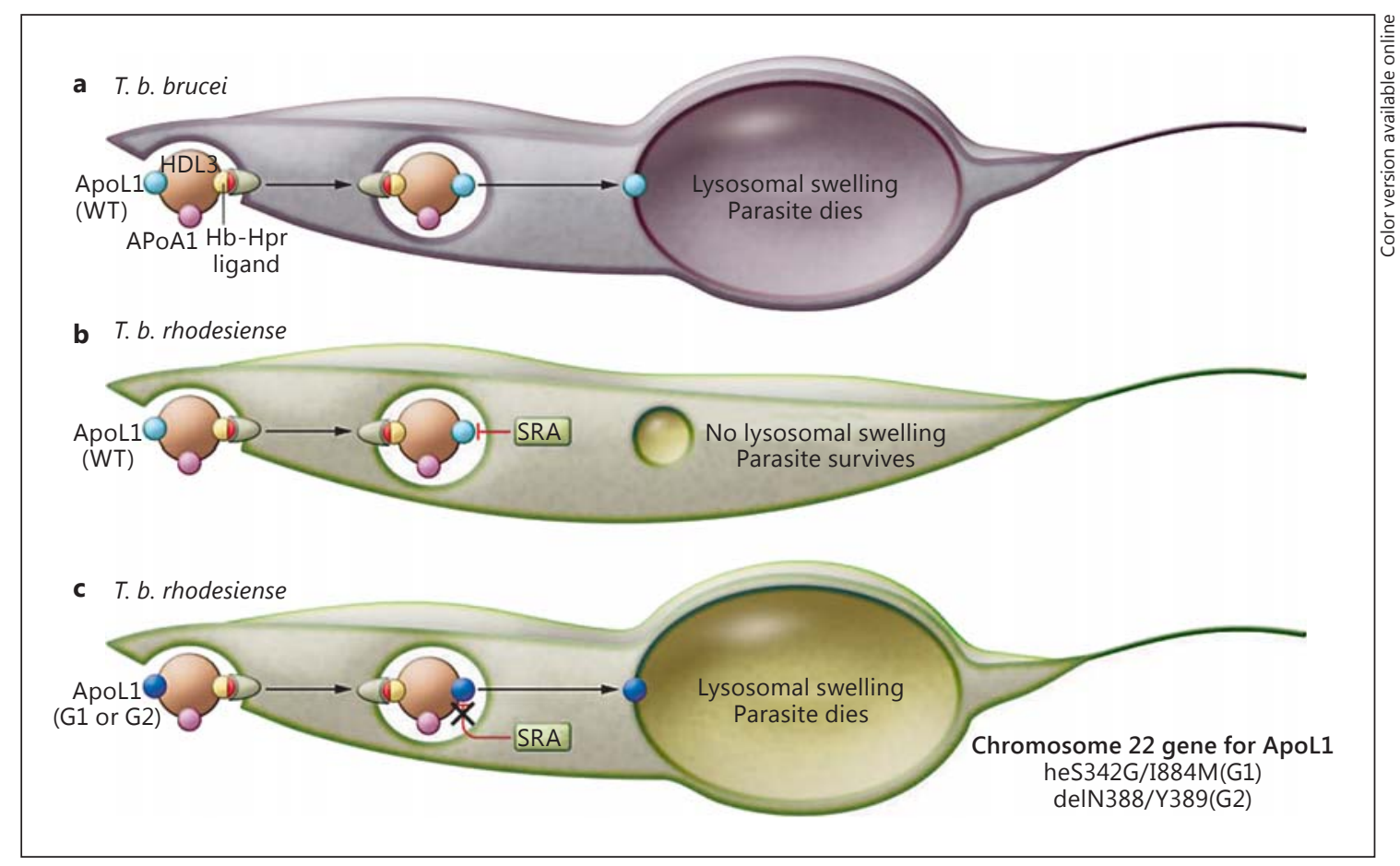

Fig. 5. Trypanolytic properties of APOL1. a Circulating APOL1 functions as a trypanosome lytic factor, forming pores in T. brucei that confer immunity against this parasite. b T. $b$. rhodesiense, which causes African sleeping sickness, can create a resistance factor called the SRA protein that can bind to the c-terminal portion of APOL1 and inhibits APOL1-mediated lysis. c APOL1 renal risk variants such as $\mathrm{G} 1$ and $\mathrm{G} 2$ variants affect a joint portion between

function as a proatherogenic, proinflammatory force rather than as a protective molecule, inhibiting or preventing atherogenesis [9].

\section{Primer on Apolipoprotein L1}

Apolipoprotein L1 (APOL1) is one of the minor apoproteins of HDL-C, newly discovered in 1997 [10]. Circulating APOL1 is a $43-\mathrm{kD}$ protein mainly found in the HDL3 subfraction and it was assumed that APOL1 might play a role in cholesterol metabolism, with no convincing evidence $[10,11]$. APOL1 is synthesized in a number of tissues, including those of the pancreas and kidney [11]. In a normal kidney, APOL1 is localized in podocytes, proximal tubules, medium-sized arteries, and arteriolar endothelial cells, but it is not certain whether localization of APOL1 in the kidney reflects de novo synthesis or uptake from circulation [11].
APOL1 and the SRA protein, prohibiting SRA binding of APOL1 and conferring resistance to infection with $T$. $b$. rhodesiense. Adapted from Friedman and Pollak [12] and Pays et al. [18] with permission from Journal of Clinical Investigation and Current Opinion in Immunology. WT = Wild type; $\mathrm{Hb}-\mathrm{Hpr}=$ hemoglobin and haptoglobin related protein.

Circulating APOL1 functions as a trypanosome lytic factor, creating pores in Trypanosoma brucei that confer immunity against this parasite [12]. T. b. rhodesiense, which causes African sleeping sickness, can produce a resistance factor called the serum resistance-associated (SRA) protein that can bind to the c-terminal portion of APOL1 and inhibits APOL1-mediated lysis (fig. 5) [12]. APOL1 renal risk variants such as G1 and G2 variants affect a joint portion between APOL1 and the SRA protein, prohibiting SRA binding of APOL1 and conferring resistance to infection with $T . b$. rhodesiense. This selective pressure was hypothesized to increase G1 and G2 variants among people of African descent and could explain the disproportionally high prevalence of nondiabetic chronic kidney disease (CKD) in people of African descent [12]. 
Fig. 6. Prevalence of ApoL1 renal risk variants among healthy and CKD populations. The high renal risk variants (2 risk variants) were prevalent among the AfricanAmerican general population in contrast to the absence of high-risk variants among the Caucasian general population. In African-Americans, the prevalence of high-renal-risk variants was higher among nondiabetic CKD and rapidly progressive CKD patients. African-Americans, patients with ESRD attributed to hypertension, and HIVAN and FSGS patients had a higher prevalence of high-renal-risk variants compared to the general African-American population. Allele frequency data was retrieved and calculated from Parsa et al. [13], Friedman et al. [19], Kopp et al. [15], and Genovese et al. [14].

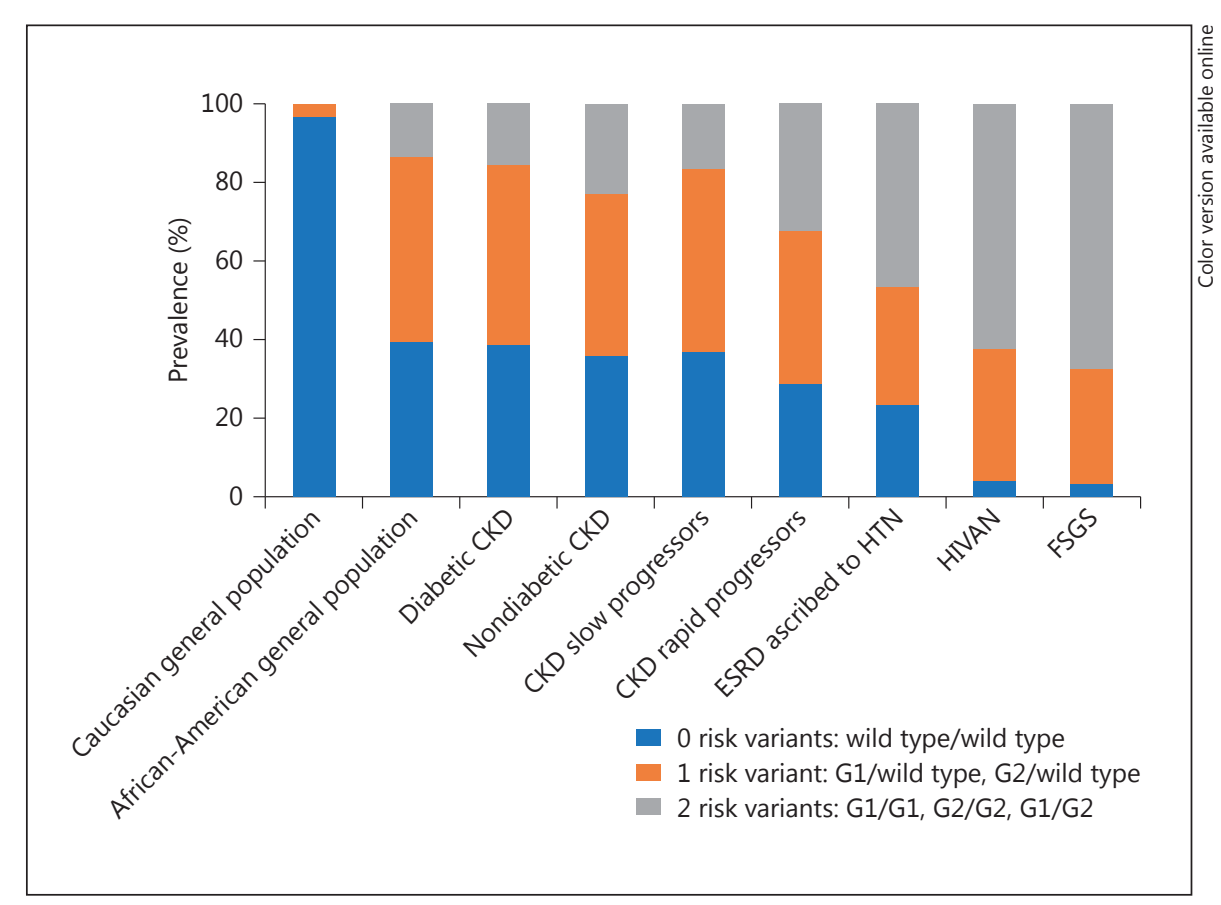

\section{Apoprotein L1 and the Progression of CKD}

APOL1 renal risk variants may explain the racial differences noted extensively in the literature between African-Americans and Caucasians with respect to the progression of CKD $[12,13]$. APOL1 renal risk variants confer 10- to 17-fold higher odds of development of focal segmental glomerulosclerosis (FSGS) [14], 29-fold higher odds of human immunodeficiency virus-associated nephropathy (HIVAN) [15], and 7.3-fold higher odds of end-stage renal disease attributed to hypertension [14].

Whether APOL1 renal risk variants affect kidney function in people with diabetes has been the subject of debate [13]. However, in the Chronic Renal Insufficiency Cohort (CRIC) study, the APOL1 high-risk variant was associated with a rapid decline in the glomerular filtration rate in black patients, regardless of the presence of diabetes mellitus [13]. In summary, analogous to how hemoglobin $\mathrm{S}$ in sickle cell disease provides protection from malaria yet causes hematologic disease over the course of a lifetime, APOL1 mutants protect against African sleeping sickness due to trypanosomiasis yet they appear to provide susceptibility to rapidly progressive CKD in those with hypertensive kidney disease, diabetes, and HIVAN and may be directly pathogenic in FSGS (fig. 6). Future research on the renal tubular catabolism of HDL and its apoproteins including APOL1 is likely to lead to new diagnostic and therapeutic targets for patients with rapidly progressive CKD.

\section{Conclusions}

The HDL-C concentration is epidemiologically associated with a reduced risk of atherosclerotic events. However, pharmacological increases in this concentration have not resulted in a change in the risk, implying that the reverse cholesterol transport has either not been altered or has been counterbalanced by some other proatherosclerotic force. APOL1 is a minor apolipoprotein which, when in mutant form, protects against trypanosome infections but which has been consistently associated with the progression of CKD in a variety of diseases. APOL1 polymorphisms may have specific relevance for explaining the racial differences noted extensively in the literature between African-Americans and Caucasians with respect to the progression of CKD and the development of end-stage renal disease. 


\section{References}

1 Gordon DJ, Probstfield JL, Garrison RJ, et al: High-density lipoprotein cholesterol and cardiovascular disease: four prospective American studies. Circulation 1989;79:8-15.

-2 Barter PJ, Caulfield M, Eriksson M, et al: Effects of torcetrapib in patients at high risk for coronary events. N Engl J Med 2007;357: 2109-2122.

-3 Schwartz GG, Olsson AG, Abt M, et al: Effects of dalcetrapib in patients with a recent Acute Coronary Syndrome. N Engl J Med 2012;367: 2089-2099.

-4 Wright RS: Recent clinical trials evaluating benefit of drug therapy for modification of HDL cholesterol. Curr Opin Cardiol 2013;28: 389-398.

5 Boden WE, Probstfield JL, Anderson T, et al: Niacin in patients with low HDL cholesterol levels receiving intensive statin therapy. $\mathrm{N}$ Engl J Med 2011;365:2255-2267.

-6 HPS2-THRIVE randomized placebo-controlled trial in 25673 high-risk patients of ER niacin/laropiprant: trial design, pre-specified muscle and liver outcomes, and reasons for stopping study treatment. Eur Heart J 2013; 34:1279-1291.
7 Trial summary of HPS2-THRIVE. 2013. http://www.cardiosource.org/Science-AndQuality/Clinical-Trials/H/HPS2-THRIVE. aspx (accessed 2014).

$>8$ Khera AV, Cuchel M, de la Llera-Moya M, et al: Cholesterol efflux capacity, high-density lipoprotein function, and atherosclerosis. N Engl J Med 2011;364:127-135.

-9 Navab M, Reddy ST, Van Lenten BJ, Fogelman AM: HDL and cardiovascular disease: atherogenic and atheroprotective mechanisms. Nat Rev Cardiol 2011;8:222-232.

10 Duchateau PN, Pullinger CR, Orellana RE, et al: Apolipoprotein L, a new human high density lipoprotein apolipoprotein expressed by the pancreas: identification, cloning, characterization, and plasma distribution of apolipoprotein L. J Biol Chem 1997;272:2557625582.

11 Madhavan SM, O’Toole JF, Konieczkowski M, Ganesan S, Bruggeman LA, Sedor JR: APOL1 localization in normal kidney and nondiabetic kidney disease. J Am Soc Nephrol 2011;22:2119-2128.

12 Friedman DJ, Pollak MR: Genetics of kidney failure and the evolving story of APOL1. J Clin Invest 2011;121:3367-3374.

-13 Parsa A, Kao WH, Xie D, et al: APOL1 risk variants, race, and progression of chronic kidney disease. N Engl J Med 2013;369:21832196.
14 Genovese G, Friedman DJ, Ross MD, et al: Association of trypanolytic ApoL1 variants with kidney disease in African Americans. Science 2010;329:841-845.

15 Kopp JB, Nelson GW, Sampath K, et al: APOL1 genetic variants in focal segmental glomerulosclerosis and HIV-associated nephropathy. J Am Soc Nephrol 2011;22:21292137.

16 Cardenas AG, et al: The importance of recognizing and treating low levels of high-density lipoprotein cholesterol: a new era in atherosclerosis management. Rev Cardiovasc Med 2007;9:239-258.

17 Brewer HB Jr, Santamarina-Fojo S: Clinical significance of high-density lipoproteins and the development of atherosclerosis: focus on the role of the adenosine triphosphate-binding cassette protein A1 transporter. Am J Cardiol 2003;92:10K-16K.

18 Pays E, et al: Human innate immunity against African trypanosomes. Curr Opin Immunol 2009;21:493-498.

19 Friedman, et al: Population-based risk assessment of APOL1 on renal disease. J Am Soc Nephrol 2011;22:2098-2105. 